\title{
Laboratory Techniques and Procedures Using Lidar
}

US Army Corps of Engineers ${ }_{\circledast}$

\section{by Gary L. Bell, Jeremy A. Sharp, Tate O. McAlpin, Anthony R. Jackson, and George B. Herring}

PURPOSE: This Coastal and Hydraulics Engineering Technical Note (CHETN) reviews the process of establishing effective and accurate techniques for the application of lidar in the laboratory with a large model domain and a need to accurately resolve issues such as local pier scour and bathymetry/topography changes. The application is for both terrestrial and line-scanning lidar.

INTRODUCTION: Lidar scanning is a process that collects high-resolution geometric, bathymetric, and topographic data. Lidar uses light pulses to conduct remote sensing and to collect high resolution data (typically referred to as a "point cloud"). Most scanners mount on a survey tripod or other fixed support structure and can rotate $360^{\circ}$ (referred to as a panoramic scan). The key component in the scanner is the polygon-shaped mirror that has flat reflective surfaces (mirror facets) arranged around a wheel perimeter to allow rotating or oscillating movement of the polygon mirror wheel (Figure 1). This allows for the measurement laser beam to be deflected, thus scanning multiple locations at once (RIEGL 2017). A brief review of lidar can be found in Jaboyedoff et al. 2010.

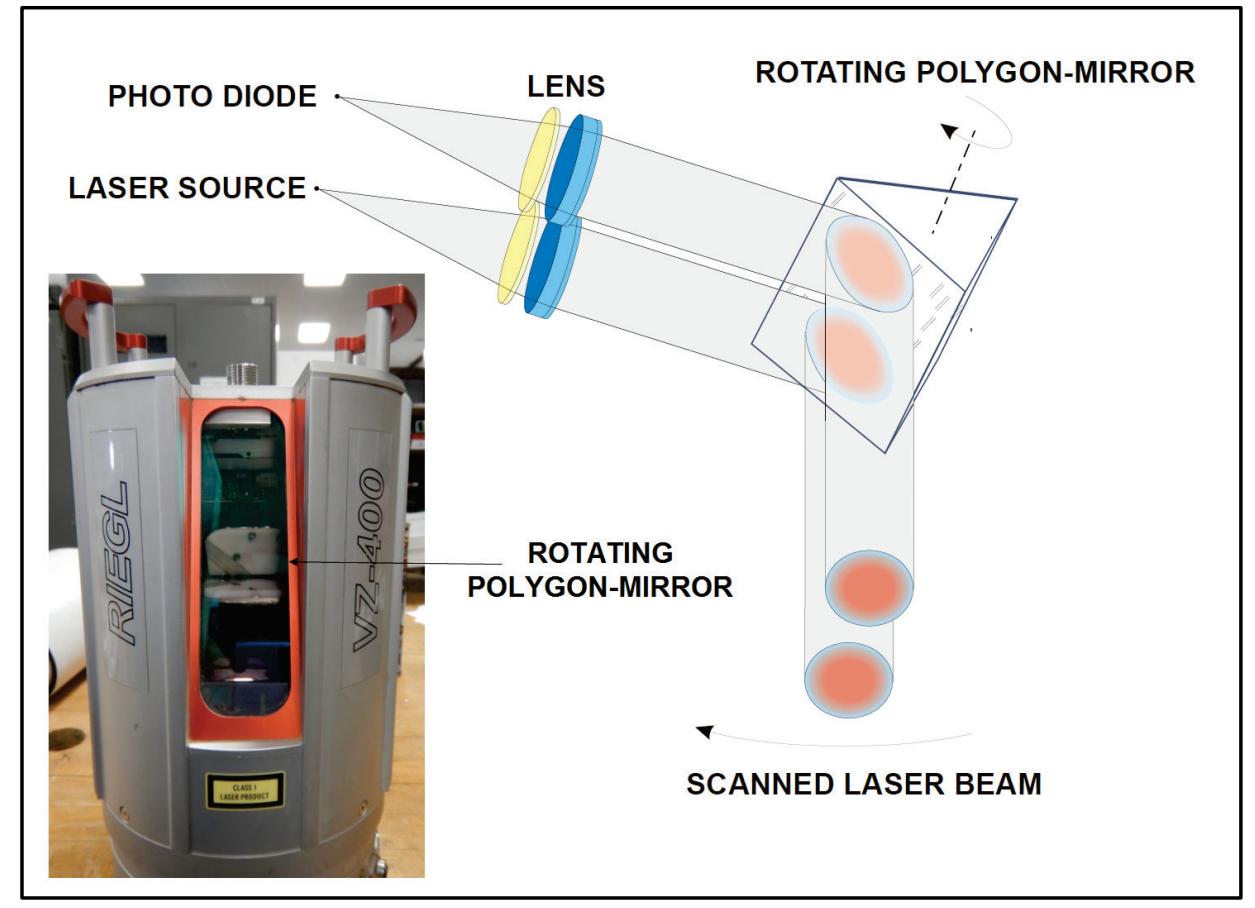

Figure 1. Diagram of the rotating polygon-mirror.

When studying sediment transport with a moveable bed model, it is advantageous to have data with high resolution and accuracy to evaluate bathymetric changes from hydraulic processes. For scaled physical models, the importance of accuracy in laboratory data collection is magnified due 
to the effects of geometric scaling. Geometry, reflectance properties of focus area, and coverage angles of point clouds are critical factors to consider for measurement accuracies (Fidera et al. 2004). For example, in regard to reflectance properties, the laser scanner generates "noise" when scanning transparent objects such as acrylic or glass. This results in generation of excess inaccurate points in that area of the point cloud. Furthermore, if the desired bathymetry were in a position that required multiple scan positions to capture all of the data, then optimization of coverage angles will benefit the user. Unlike the challenges of traditional bathymetric data collection methods, the high resolution of terrestrial scanning provides complete coverage of the domain. The proper planning and use of a lidar system provides an accuracy of millimeters, minimizing measurement error that is magnified when scaled to prototype dimensions.

Traditional methods of surveying such as total station, automatic level, and sliding elevation rods might have equivalent levels of accuracy but lack the resolution. These methods, while representing different levels of technology, provide data at one point and require the user to physically move the measurement instrument between data points. Additionally, traditional methods require a multi-person crew to collect data whereas one individual can operate a lidar scanner and collect millions of data points in a matter of minutes. In addition to a $360^{\circ}$ panoramic scan, some lidar systems can also operate in a line-scan mode. A line scan is a high-detail set of X $\mathrm{Y} Z$ data that is taken along a single line at a rapid rate. This produces high-resolution data at a single cross section or profile. Performing a series of line scans produces a temporal set of profiles. Line scanning is advantageous for coastal models where nearshore waves expose coastal features for brief periods of time. Note that there are technologies such as green lasers that can penetrate the water surface and collect data; however, the laser power required to do this at the laboratory scale is great, which generates extremely dangerous conditions.

TERRESTRIAL SCAN: Terrestrial scanning (also known as three-dimensional [3D] scanning) is the collection of topographic or bathymetric data over a given area. The terrestrial scanning technique described here was developed from the procedure established for the 1:30 scaled model of the Burlington Northern Santa Fe Corporation railroad crossing on the Santa Ana River near Corona, CA. This same technique has been successfully applied to four other models: Mud Mountain Fish Barrier Section Model, Mud Mountain Fish Barrier General Model, Large-Scale Sediment Transportation Facility (LSTF) dredge material placement, and Vegetative Dune Overtopping Study. The Santa Ana River model had tremendous lidar challenges. These included a large model domain $45 \mathrm{feet}(\mathrm{ft}$ ) wide and $108 \mathrm{ft}$ long and steep scour holes (see Figure 2). The study's focus is pier scour, so high resolution and accuracy were required around the bridge piers (Figure 2). For accurate lidar scanning of a large moveable bed model, there are necessary steps to follow. Those steps are outlined in the following text.

First, 21 white, reflective targets were set up around the model (Figure 3 shows a subset of the target locations). Two types of reflectors were used: flat, 5-centimeter $(\mathrm{cm})$ diameter targets and $2.5 \mathrm{~cm}$ cylindrical $(2.5 \mathrm{~cm}$ height and width) targets. The placement of these targets is important. Since lidar is line-of-sight limited and with a large model domain in conjunction with steep bathymetry, multiple scan positions were required. The flat targets provided a bigger (and thus easier) surface to acquire position and were useful for long-range positioning (i.e., locating targets at the opposite end of the hangar). However, for the best results it is ideal to scan the flat targets exactly perpendicular. This leads to higher accuracies when tying in the multiple scan positions due to the accurate scan of the targets' size and location. The $2.5 \mathrm{~cm}$ cylindrical targets can be 
captured in their entirety from a wide variety of positions and do not require an exact perpendicular line-of-sight when scanning them. However, due to the smaller size of the targets, they are more difficult to locate with increased distance as well as slightly less accurate at these farther-away locations. For each scan position there should be multiple targets at varying elevations, which helps tie the data together in the same plane. The positions of all possible scanning locations were identified prior to the placement of the targets. Initial mapping/locating of the scan positions was done by visual inspection of the model, which was confirmed by actual lidar scanning. By locating scanning positions prior to collection, the target placement is verified to satisfy the above requirements. Scanner positioning is critical to ensure complete coverage of the area of interest, the level of data resolution, and the accuracy obtained when combining multiple scan positions during the data processing stage.

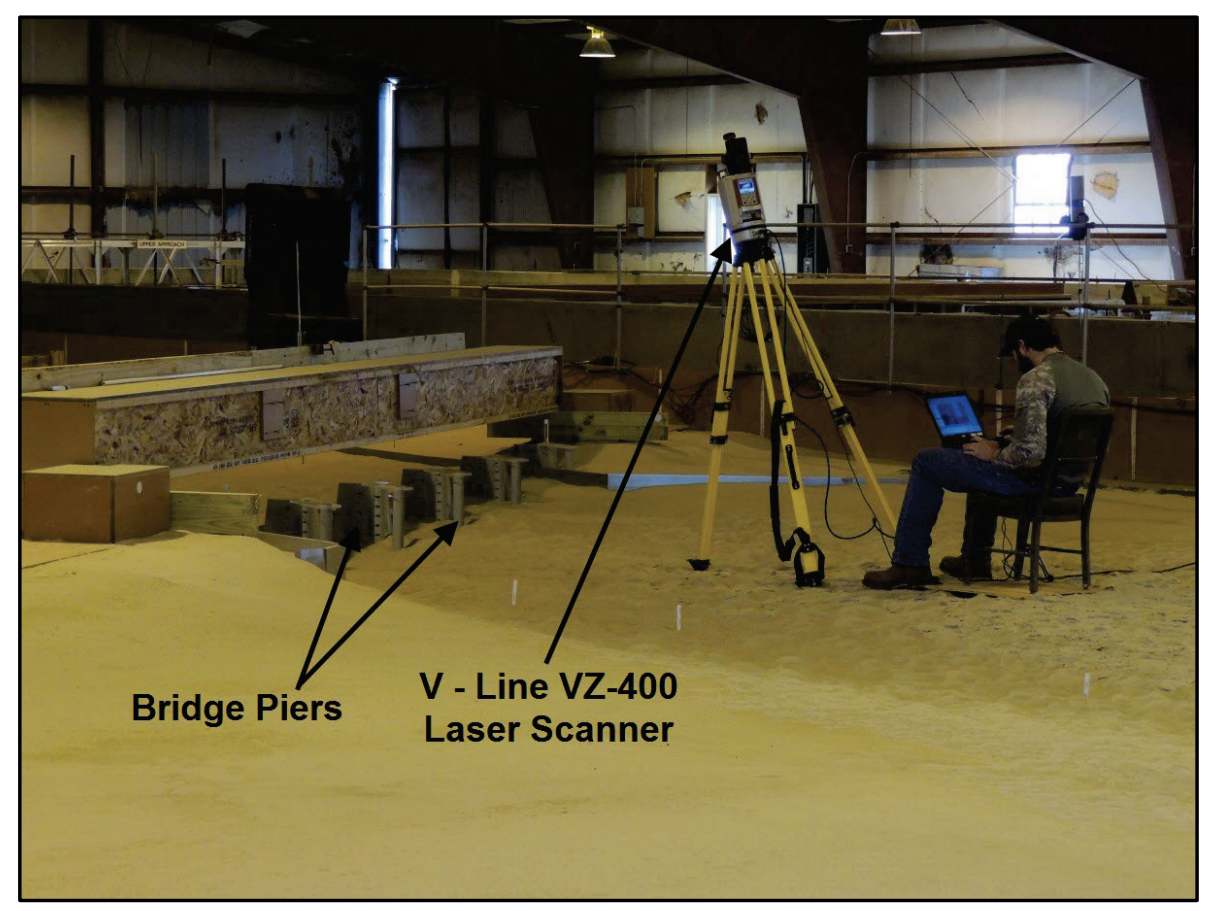

Figure 2. Conducting a post-test lidar scan.

A local coordinate system is used to define the location of each target, which allows the user to combine multiple scan positions and tests so that all are projected correctly. Of the 21 targets, 13 were surveyed into a local coordinate system using a Nikon total station. This target survey was then used to define the Global Coordinate System (GLCS) in the RIEGL software, which in turn was then used to tie the multiple scan positions and tests to the same global plane. It is recommended when laying out the GLCS, to define a "zero" or reference line for proper model orientation. For example, orientating the longitudinal length as the y-axis is beneficial for orientation in processing. Note that any physical movement of these control points will result in increased error. The location of the control points in the model can be seen in Figure 4 (the view is looking at the entire model from a position upstream). All unnecessary data (e.g., hangar walls) have been removed, and the view is above the model looking downstream. 


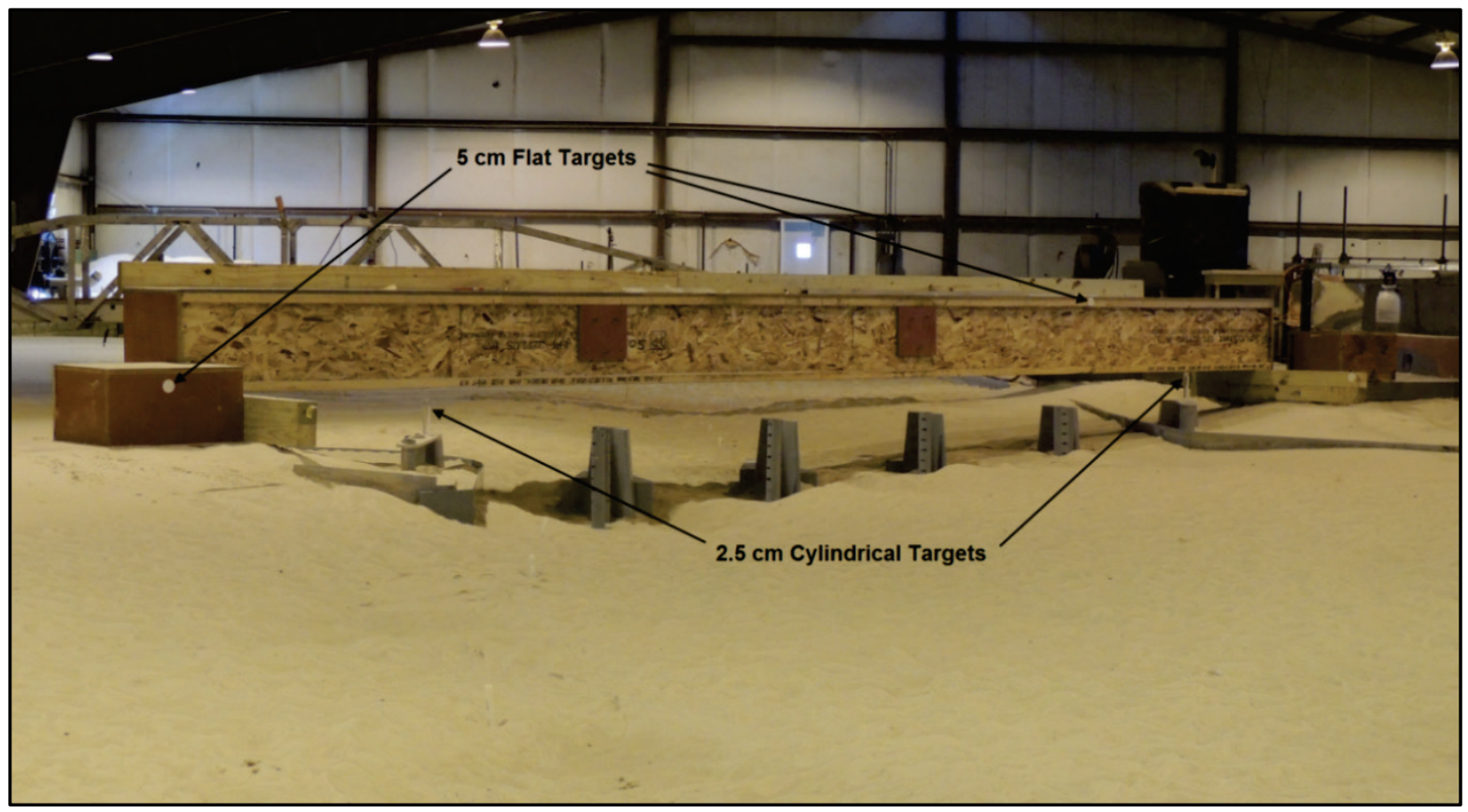

Figure 3. Reflective targets.

Next, terrestrial scans were performed for both pre- and post-model testing. For the Santa Ana Model, a RIEGL V - Line VZ-400 (V: Online Waveform Processing; Z: Z-axis) terrestrial laser scanner was used (Figure 1). The accuracy of this instrument is approximately $5^{\circ}$ millimeters $(\mathrm{mm})$ horizontal and vertical $(0.15$ meter [m] prototype for the Santa Ana Model). The vertical scan angle range is up to $100^{\circ}\left(+60^{\circ} /-40^{\circ}\right)$. This vertical scan angle range also plays a role in determining the amount of data that will be taken with each scan position as the area is limited by the vertical angle constriction. In general, approximately six scans at different scan positions were done pre-test and eight positions post-test. The increase in scan positions between pre-test and post-test was due to line-of-sight limitation in conjunction with the steep scour holes around the piers. Number and location of scan positions will vary based on the model objective, configuration, and size. In general, the fewer scans that are needed to collect the necessary data, the more efficiency in post-processing the data (smaller file sizes, less time collecting the data, decreased data processing time, etc.). However, if there is uncertainty in how much data and/or the level of resolution is needed, then taking more scans is advantageous. Figure 5 displays post-test lidar data with multiple scan positions combined in one view (different colors represent separate scan positions). The scans can be deleted/trimmed in the future during the data processing stage. Figure 6 is the result of a pre-test minus post-test analysis after the data have been processed. 


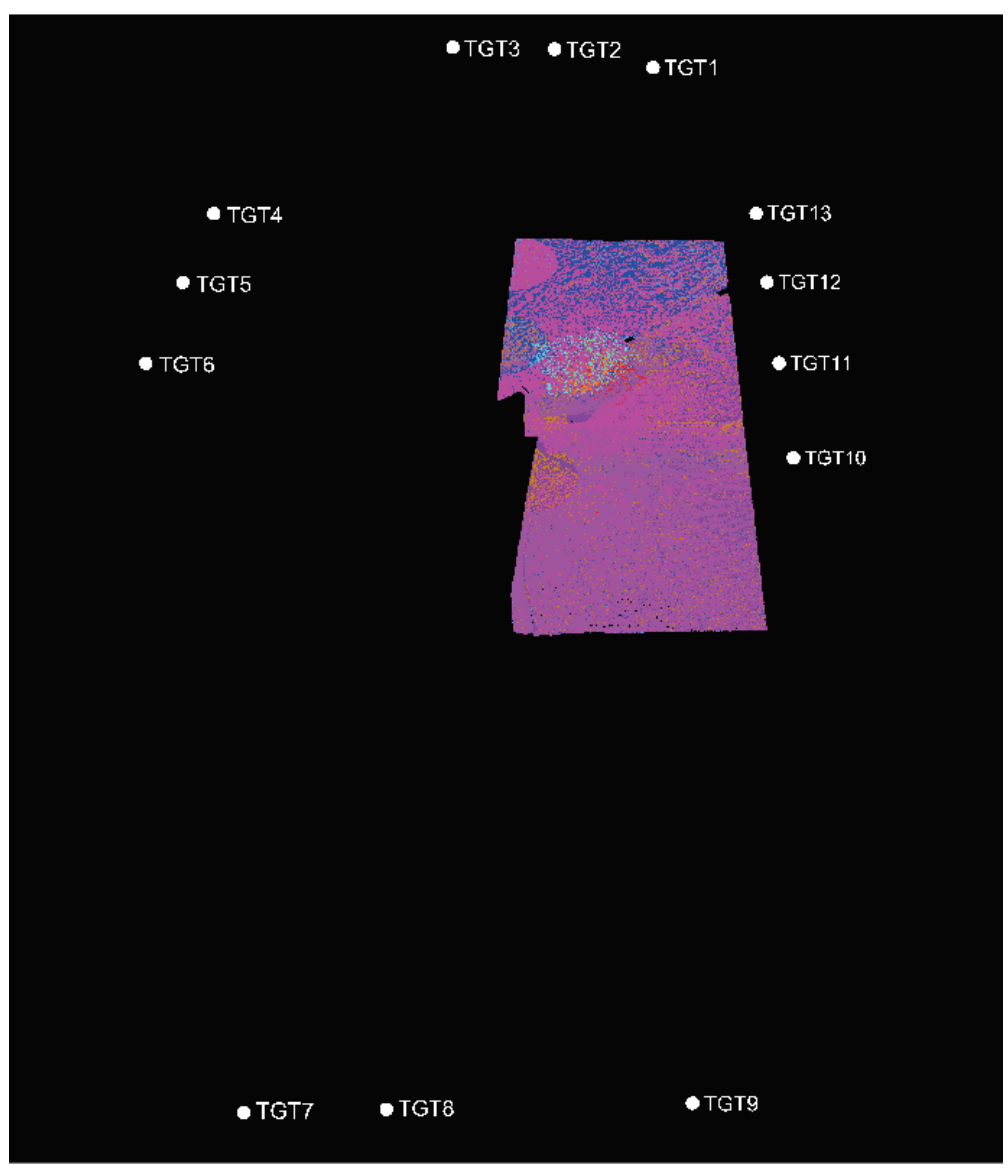

Figure 4. Control targets.

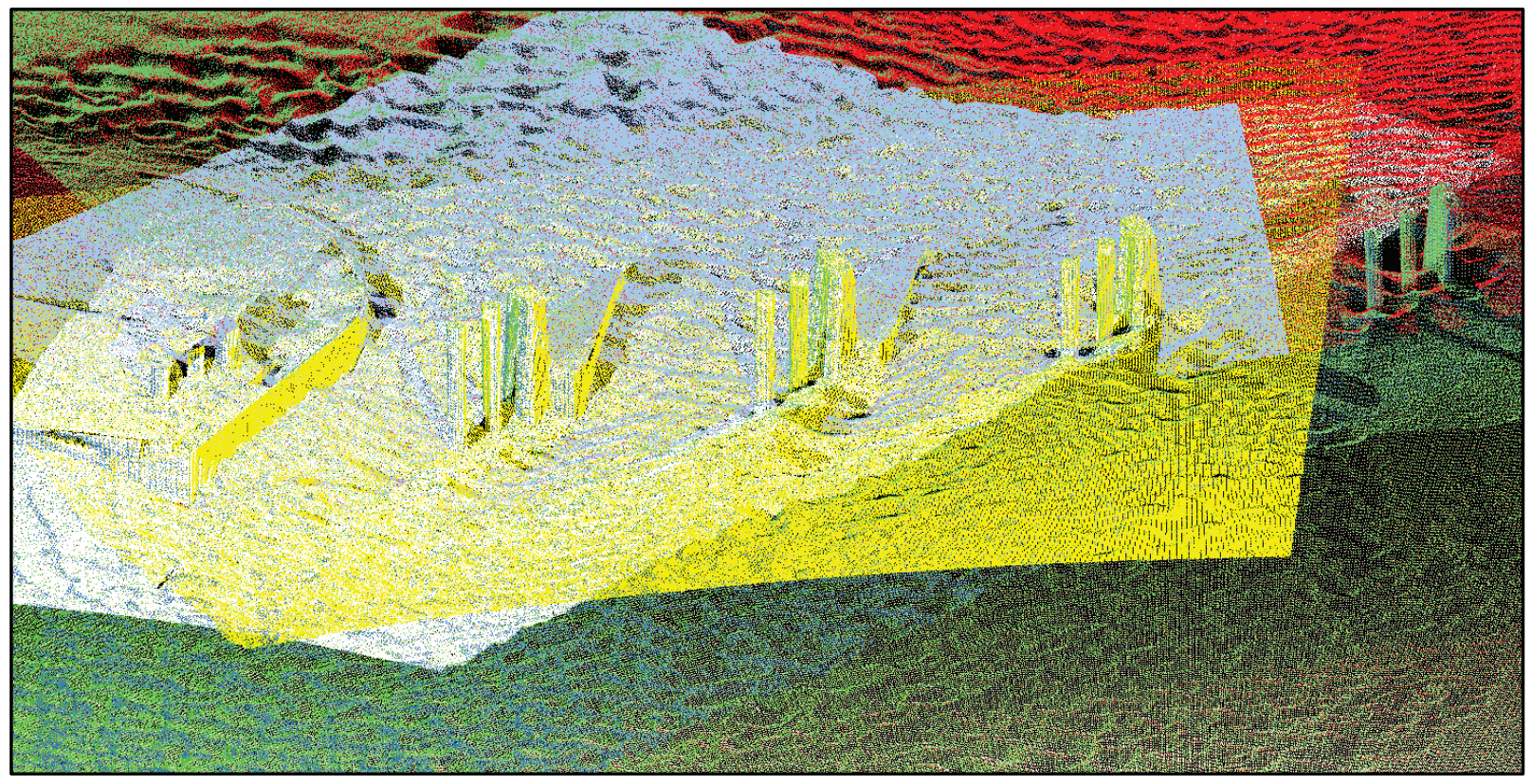

Figure 5. Post-test lidar after data processing in RiSCAN PRO (looking upstream at piers $6-2$, starting from the left). 


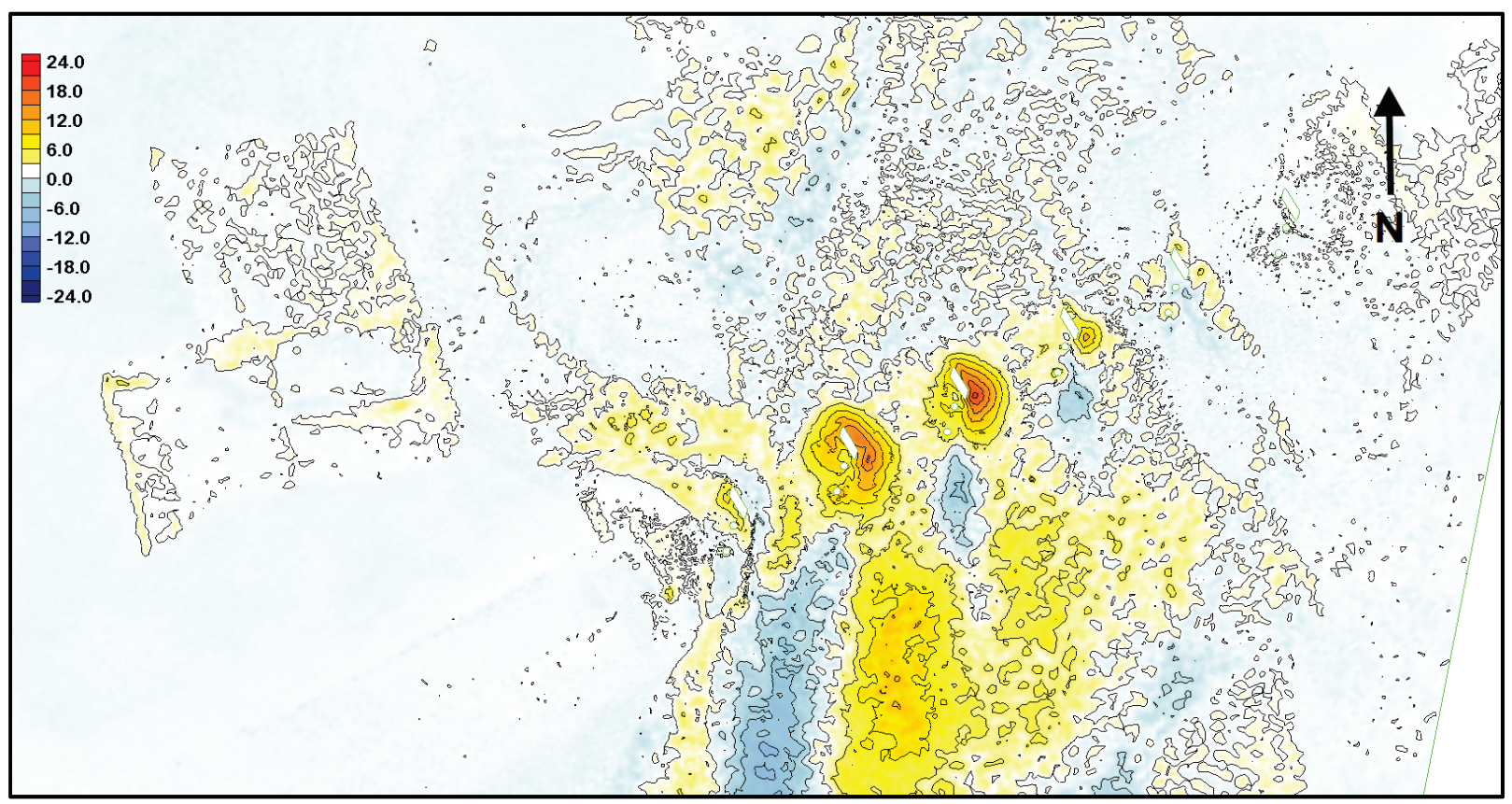

Figure 6. Plan view pre-test minus post-test results (in feet, prototype).

LINE SCAN: As previously mentioned, some lidar scanners can also be used in line-scan mode. Line scanning requires the same process as above to establish the scans globally. Here, the procedure established for line scanning was formulated from the Vegetative Dune Overtopping Study. The Vegetative Dune Overtopping study was conducted in a $1.52 \mathrm{~m}$ wide wave flume located at the U.S. Army Engineer Research and Development Center (ERDC), Coastal and Hydraulics Laboratory (CHL), in Vicksburg, MS (Figure 7). The model was a 1:15 scale movable bed model of a typical beach and dune geometry. The focus of the study was the analysis of erosion from waves colliding and overtopping a vegetated dune. The instrument used for terrestrial scans and line scans was the REIGL LMS-Z390(i) laser scanner. The accuracy of this instrument is approximately $6 \mathrm{~mm}$ horizontal and vertical (0.09 m prototype). The instrument was mounted above the wave flume in such a way that it could collect both types of data sets: line scans and panoramic scans (Figure 7).

Line scanning allows for data to be taken within the instruments' vertical angle boundaries (for the LMS-Z390(i) the vertical scanning range is up to $80^{\circ}$ ) while staying in the same horizontal location $\left(0^{\circ}\right.$ to $360^{\circ}$ horizontally). The scan rate, resolution, and amount of scans can be adjusted to suit model-specific requirements. In Figure 8, the panoramic 3D lidar-scan data (fuchsia) and the linescan data (aqua) are displayed on top of each other. The line-scan data were taken temporally to track the progression of dune erosion. The scanning rate is 10 scans per second. Also displayed is the LMS-Z390(i) scan position location relative to the collected data set along with the representative Cartesian axes for reference (x-axis is red, y-axis is green, and $\mathrm{z}$-axis is blue). This image is only representing one of several thousand frames collected for each test. Note that the line-scan data point size is not representative of the horizontal scan angle; the size was increased for visualization purposes only. Furthermore, the line-scan data is constrained to the length shown in Figure 8 due to water in the model. 
Similar to the previously mentioned data, the line-scan data can be analyzed and manipulated by the use of MATLAB, RiSCAN PRO, and many other analysis tools. This application also records a timestamp on the data so that a point can be analyzed at any specific time during the model simulation.

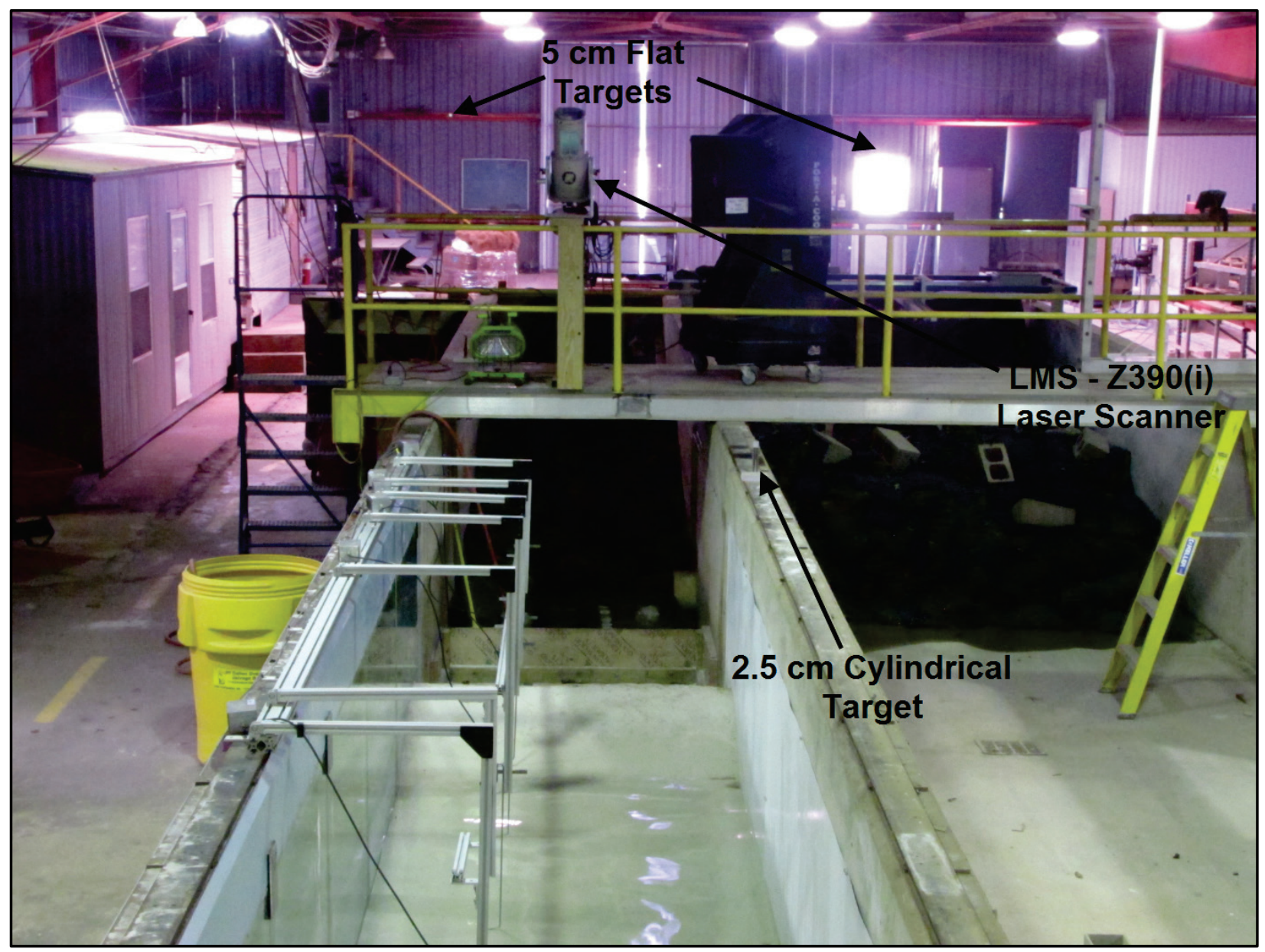

Figure 7. Flume study, ERDC-CHL, Vicksburg, MS. 


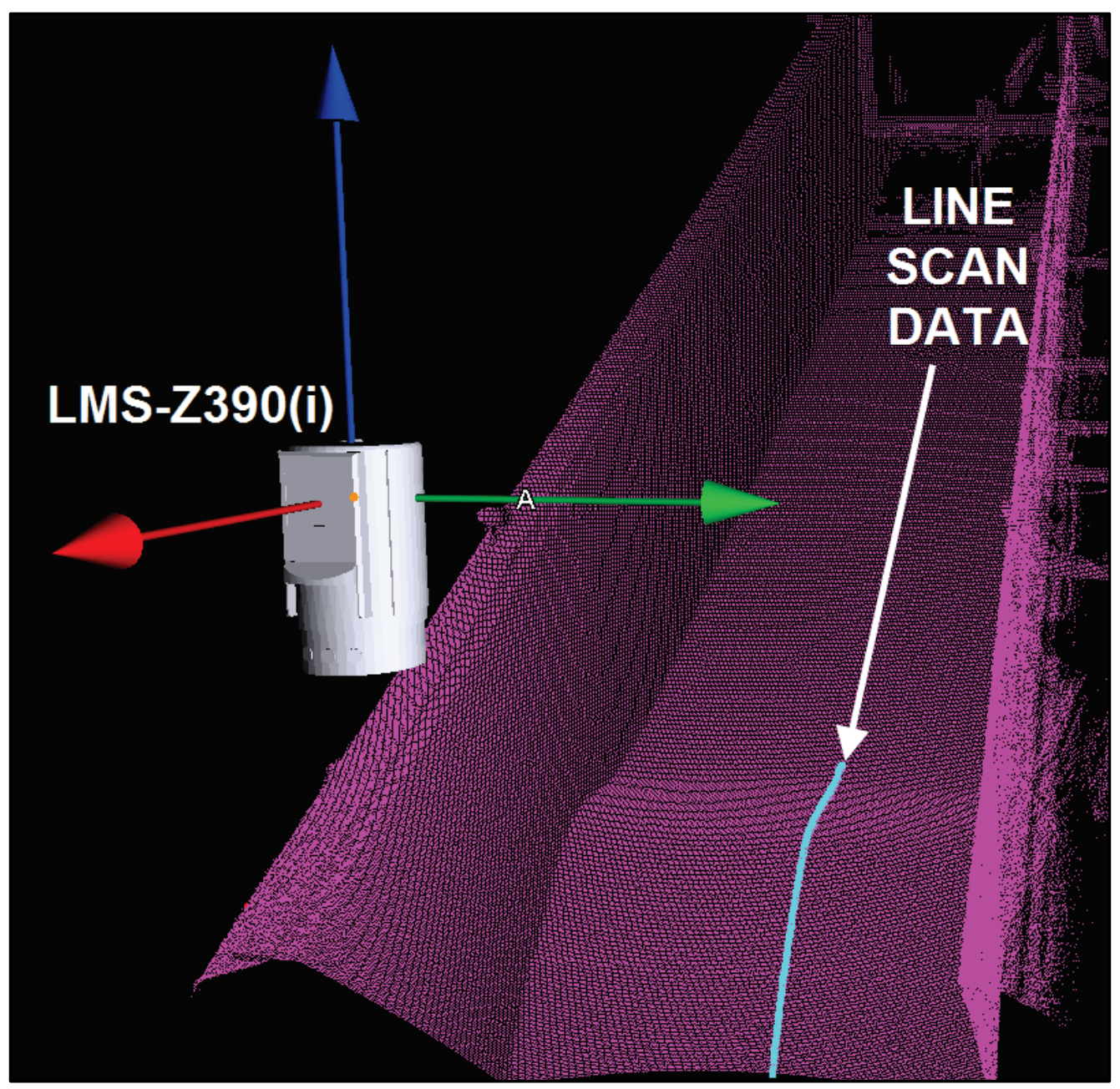

Figure 8. Line-scan data from the LMS - Z390(i).

PROCESSING DATA: The collected lidar data were processed using RiSCAN PRO software (Figure 5 and 8) (RiSCAN PRO 1.7.4). This software allowed for the import of the control points established using the total station. After the lidar data were processed and exported as XYZ data, a MATLAB code was applied to scale, rotate, and translate the point cloud data to prototype coordinates and units. The data were then taken and interpolated onto a two-dimensional grid using SMS (Surface Water Modeling System) software. With the grid at prototype coordinates, units, and scale, a PRE minus POST (pre-test minus post-test) analysis could be performed. In MATLAB, the POST mesh was subtracted from the PRE mesh to obtain a difference mesh. The result illustrates scour and deposition locations and can also be used to determine scour/deposition volumes (volumes not present in this study) (Figure 6). The PRE to POST comparisons of the processed lidar data averaged approximately $+/$ - error of $5 \mathrm{~mm}$ (approximately $0.15 \mathrm{~m}$ prototype). The SMS software package was used to view the PRE and POST model results translated to the prototype location. This allowed for visual representation of scour depths at the prototype locations, quantitative comparisons of the scour/deposition areas in the model, and difference plots between the PRE and POST model simulations (Figure 6). 
SUMMARY: The importance of high resolution and accuracy is evident when studying sediment transport in a moveable-bed scaled physical model. Using terrestrial lidar scanning as a form of data collection and analysis of model results has increased the accuracy/efficiency of physical modeling in the following ways:

1. A significant increase in gathered information regarding $\mathrm{X} Y \mathrm{Z}$ data.

2. Increased accuracy in measurements.

3. Increased speed of data collection.

4. Supplemented physical model conclusions from gathered data using numerical modeling.

5. Accurate volumetric transformation calculations from pre to post test.

6. Controllable coordinate systems which can be projected on to prototype locations.

7. Conventional forms of data (i.e., text files) that can be used in multiple ways.

8. Time-monitored progression of a fluctuating characteristic in the model using line scan.

CONCLUSION: Panoramic and/or line-scan lidar applications have the capability to increase specific detail to physical model data results. This application results in higher accuracies in measurements and collected data, increased efficiency, and an expanded collection of analysis techniques. These qualitative and quantitative advances in physical modeling are beneficial to the engineer/scientist in multiple ways, specifically in the resulting data analysis. Possible advances in this category of technology are apparent. Three-dimensional laser tracking (position and orientation) could be deployed for real-time laboratory data collection, measurements, structure placement, etc. Flash lidar has the ability to collect topographical mapping data more rapidly than point-scan technology (decreasing scan time); it is light weight and generally immune to motionrelated scan errors due to the speed-of-light data capture; it has full frame time-of-flight data (3D image) collected with a single laser pulse; and it possesses high accuracy combined with technology that is capable of recording the required sequential temporal information (Roback et al. 2013). Green laser technology (with some adjustments for safety and practicality) has the potential to be deployed in the laboratory for sub-aqueous measurements. These combinations of lidar/laser technology are obvious candidates for future physical model application development.

ADDITIONAL INFORMATION: This Coastal and Hydraulics Engineering Technical Note (CHETN) was prepared by Gary L. Bell, research hydraulic engineer at the U.S. Army Engineer Research and Development Center, Coastal and Hydraulics Laboratory, Vicksburg, MS. Questions about this Technical Note can be addressed to Mr. Bell at 601-634-4621 or Gary.L.Bell@usace.army.mil.

Gary L. Bell, Jeremy A. Sharp, Tate O. McAlpin, Anthony R. Jackson, and George B. Herring. 2018. Laboratory Techniques and Procedures Using Lidar. ERDC/CHL CHETN-VII-18. Vicksburg, MS: U.S. Army Engineer Research and Development Center. http://dx.doi.org/10.21079/11681/26288 


\section{REFERENCES}

Alexander Bulyshev; Farzin Amzajerdian and Robert Reisse. 2013."Helicopter Flight Test of 3D Imaging Flash LIDAR Technology for Safe, Autonomous, and Precise Planetary Landing." In Proc. SPIE 8731, Laser Radar Technology and Applications XVIII, 87310H, June 4, 2013. doi:10.1117/12.2015961; http://dx.doi.org/10.1117/12.2015961.

Fidera, Artur, Michael A. Chapman, and Jingook Hong. 2004. XXth ISPRS Congress, 12-23 July 2004 Istanbul, Turkey, Technical Commission V, Volume XXXV Part B5, 880-883. http://www.isprs.org/proceedings/XXXV/congress/ comm5/papers/673.pdf.

Jaboyedoff, Michel, Thierry Oppikofer, Antonio Abellán, Marc-Henri Derron, Alex Loye, Richard Metzger, and Andrea Pedrazzini. 2012. "Use of LIDAR in Landslide Investigations: A Review." Natural Hazards 61(1): 5-28.

RIEGL. 2017. 3D Terrestrial Laser Scanner: RIEGL VZ-400, RIEGL VZ-1000, RIEGL VZ-2000: General Description and Data Interfaces. Horn, Austria: RIEGL Laser Measurement Systems GmbH.

NOTE: The contents of this technical note are not to be used for advertising, publication, or promotional purposes. Citation of trade names does not constitute an official endorsement or approval of the use of such products. 